\title{
Noncollisional kinetic model for non-neutral plasmas in a Penning trap: General properties and stationary solutions
}

\author{
G. G. M. Coppa* and Paolo Ricci \\ Istituto Nazionale per la Fisica della Materia (INFM) and Dipartimento di Energetica, Politecnico di Torino, \\ Corso Duca degli Abruzzi 24, 10129 Torino, Italy \\ (Received 6 May 2002; published 15 October 2002)
}

\begin{abstract}
This work deals with a noncollisional kinetic model for non-neutral plasmas in a Penning trap. Using the spatial coordinates $r, \theta, z$ and the axial velocity $v_{z}$ as phase-space variables, a kinetic model is developed starting from the kinetic equation for the distribution function $f\left(r, \theta, z, v_{z}, t\right)$. In order to reduce the complexity of the model, the kinetic equations are integrated along the axial direction by assuming an ergodic distribution in the phase space $\left(z, v_{z}\right)$ for particles of the same axial energy $\epsilon$ and the same planar position. In this way, a kinetic equation for the $z$-integrated electron distribution $F(r, \theta, \epsilon, t)$ is obtained taking into account implicitly the three-dimensionality of the problem. The general properties of the model are discussed, in particular the conservation laws. The model is also related to the fluid model that was introduced by Finn et al. [Phys. Plasmas 6, 3744 (1999); Phys. Rev. Lett. 84, 2401 (2000)] and developed by Coppa et al. [Phys. Plasmas 8, 1133 (2001)]. Finally, numerical investigations are presented regarding the stationary solutions of the model.
\end{abstract}

DOI: 10.1103/PhysRevE.66.046409

PACS number(s): 52.25.Dg

\section{INTRODUCTION}

Non-neutral plasmas are commonly confined by using a Penning-Malmberg trap. The device [1-3] consists of a conducting cylinder of radius $R_{w}$, divided axially into three sections. The central section, which extends from $z=-L_{c} / 2$ to $z=L_{c} / 2$, is grounded, while the end electrodes (each having length $L_{s}$ ) are at a negative potential $-V$ for electron plasmas. The plasma is mainly confined in the central section, the axial confinement being provided by the electrostatic fields and the radial confinement by a uniform axial magnetic field $\mathbf{B}=B_{0} \mathbf{e}_{z}$.

Usually, the dynamics of these plasmas is described by employing a two-dimensional drift-Poisson model, where charged particles are regarded as straight lines of uniform density, due to the very high value of the axial bouncing frequency with respect to all the other frequencies of interest $[4,5]$. According to the standard model, the particles exhibit an $\mathbf{E} \times \mathbf{B}$ drift motion and the electric field is computed selfconsistently from the charge density by using the Poisson equation. Consequently, the plasma evolves as a twodimensional inviscid fluid; in fact, the drift-Poisson model is isomorphic to the two-dimensional Euler equations for a uniform density fluid [6,7]: in particular, a perfect analogy exists between the Kelvin-Helmholtz instability and the $\mathbf{E} \times \mathbf{B}$ diocotron instability for a plasma [4]. Using a linear theory, possible instabilities are predicted for the azimuthal modes $m_{\theta}>1$, while the $m_{\theta}=1$ mode is shown to be stable for any density profile $[4,5]$. Also, it has been shown that the continuous eigenvalue spectrum of the mode $m_{\theta}=1$ produces only an algebraic growth of an initial perturbation [8]. Nevertheless, experiments show that the mode growth is actually exponential $[3,9]$. The contradiction between experimental results and linear two-dimensional theory is a challenging

\footnotetext{
*Email address: ggmcoppa@polito.it
}

problem in the theory of non-neutral plasmas.

Recent studies on the evolution of non-neutral plasmas in a Penning-Malmberg trap have pointed out the important role of kinetic effects and of the finite length of the device, in particular for the $m_{\theta}=1$ diocotron instability [10-13]. In fact, when a particle approaches the end of the plasma, it experiences a confining end potential which depends on both the radius $r$ and the axial coordinate $z$. The radial component of the confining electric field causes an $\mathbf{E} \times \mathbf{B}$ drift in the azimuthal direction and the rotation frequency is affected, depending on the axial energy of the particles. In fact, the axial energy affects the penetration length in the confining potential and, as a consequence, the radial electric field the particle experiences [14-17].

The aim of the present work is to develop a self-consistent kinetic theory for non-neutral plasmas in a PenningMalmberg trap in order to take into account self-consistently all these effects. A preliminary report of this kinetic theory was published in Ref. [18].

The kinetic model assumes $[9,19]$ that the frequencies are ordered as $\omega_{c} \gg \omega_{b} \gg \omega_{E}, \omega_{c}$ being the cyclotron frequency, $\omega_{b}$ the bouncing frequency, and $\omega_{E}$ the $\mathbf{E} \times \mathbf{B}$ drift rotation, which is comparable to the frequency of the diocotron modes. Moreover, the scale lengths are assumed to be such that $R_{w} \gg \lambda_{D} \gg r_{c}$, where $R_{w}$ represents the typical macroscopic dimension of the plasma, $\lambda_{D}$ is the Debye length, and $r_{c}$ is the cyclotron radius. With these assumptions, the planar motion of the electrons is described by the dynamics of their guiding centers, given by the $\mathbf{E} \times \mathbf{B}$ drift velocity, and a complete kinetic description of the plasma is provided by the distribution function $f\left(r, \theta, z, v_{z}, t\right)$. The description is completed by the self-consistent Poisson equation for the electrostatic potential $\phi(r, \theta, z, t)$.

To obtain a model that can be dealt with, both analytically and numerically, the dimensionality of the problem is reduced, by integrating along the axial direction. Considering that the electrostatic potential experienced by a string of 
electrons varies slowly in time, an ergodic distribution in the phase space $[20,21]$ can be assumed for particles of the same "axial energy," $\epsilon$, defined as the sum of axial kinetic and potential energies, and the same planar position $(r, \theta)$, in order to obtain a kinetic equation for the $z$-integrated electron distribution $F(r, \theta, \epsilon, t)$. Such a kinetic equation is the main subject of the present work.

The paper is organized as follows. The kinetic equation for $F(r, \theta, \epsilon, t)$ is deduced in Sec. II. Section III analyzes conservation laws for the model, while Sec. IV deals with the fluid equation of the plasma: the fluid equations presented in Ref. [11] can be deduced from the kinetic model assuming a Maxwellian distribution for the axial energy of the particles, $\epsilon$. Section $\mathrm{V}$ deals with the properties of the distribution function $F(r, \theta, \epsilon, t)$. Finally, in Sec. VI numerical results on the equilibrium configurations are presented.

\section{BASIC EQUATIONS}

As $\omega_{c} \gg \omega_{b} \gg \omega_{E}$ and $R_{w} \gg \lambda_{D} \gg r_{c}[9,19]$, the guiding center drift approximation can be used to describe the dynamics of the plasma particles. According to this assumption, the planar velocity of the particles is fixed by the $\mathbf{E} \times \mathbf{B}$ drift. Thus, the set of coordinates $\left(r, \theta, z, v_{z}\right)$ provides a full description of the state of a particle [17], and a complete kinetic representation of the plasma is given by the distribution function $f\left(r, \theta, z, v_{z}, t\right)$, whose dynamics is governed by the following kinetic equation:

$$
\begin{aligned}
\frac{\partial f}{\partial t}+ & \nabla_{\perp} \cdot\left(\frac{\mathbf{e}_{z} \times \nabla_{\perp} \phi}{B_{0}} f\right)+\frac{\partial}{\partial z}\left(v_{z} f\right) \\
& +\frac{\partial}{\partial v_{z}}\left(\frac{e}{m} \frac{\partial \phi}{\partial z} f\right)=0 .
\end{aligned}
$$

The kinetic equation (1) and the Poisson equation

$$
\nabla^{2} \phi=\frac{e}{\varepsilon_{0}} \int f\left(r, \theta, z, v_{z}, t\right) d v_{z}
$$

constitute the complete kinetic model for a non-neutral plasma in a Penning trap.

This model is very complex to treat either analytically or numerically. Also, the information it provides is too rich, as experimental measurements do not yield $f\left(r, \theta, z, v_{z}, t\right)$ directly, but the $z$-integrated distribution of the particle, $F(r, \theta, \epsilon, t)$, where $F(r, \theta, \epsilon, t) \Delta \epsilon$ denotes the number of particles per unit of area $(r, \theta)$ at time $t$, with axial energy between $\epsilon$ and $\epsilon+\Delta \epsilon$; the axial energy of the particles, $\epsilon$, being defined as the sum of axial kinetic and potential energies:

$$
\epsilon=\frac{1}{2} m v_{z}^{2}-e \phi(r, \theta, z, t)
$$

In general, the relation between the distribution functions $f\left(r, \theta, z, v_{z}, t\right)$ and $F(r, \theta, \epsilon, t)$ can be expressed as

$$
f\left(r, \theta, z, v_{z}, t\right)=\int F(r, \theta, \epsilon, t) G\left(z, v_{z}, r, \theta, \epsilon,\{\phi\}, t\right) d \epsilon,
$$

where $G$ expresses the probability of finding a particle with energy $\epsilon$ and with planar position $(r, \theta)$ at the axial position $z$ and the velocity $v_{z}$. The function $G$ is a functional of the trap potential $\phi$ and, according to the definition of probability, $G$ is normalized in such a way that $\iint G d z d v_{z}=1$.

Denoting by $H$ the axial Hamiltonian of a particle [20],

$$
H=\frac{1}{2} m v_{z}^{2}-e \phi(r, \theta, z, t)
$$

the kinetic equation for the distribution function $F$ can be deduced from Eq. (1), assuming [21] that the particles with $H=\epsilon$ are distributed according to the ergodic hypothesis [i.e., uniformly in the phase space $\left.\left(z, v_{z}\right)\right]$ so that $G$ can be expressed as

$$
\begin{aligned}
G\left(z, v_{z}, r, \theta, \epsilon,\{\phi\}, t\right) & =\frac{\delta(H-\epsilon)}{\iint \delta(H-\epsilon) d z d v_{z}} \\
& =\frac{\delta(H-\epsilon)}{J(r, \theta, \epsilon,\{\phi\}, t)},
\end{aligned}
$$

where

$$
\begin{aligned}
J(r, \theta, \epsilon,\{\phi\}, t) & =\iint \delta(H-\epsilon) d z d v_{z} \\
& =\int \sqrt{\frac{2}{m[\epsilon+e \phi(r, \theta, z, t)]}} d z
\end{aligned}
$$

In order to deduce the kinetic equation for $F(r, \theta, \epsilon, t)$, an arbitrary function of the Hamiltonian is introduced, $\psi(H)$, and the macroscopic quantity $\Psi(r, \theta, t)$ is defined as

$$
\Psi(r, \theta, t)=\iint \psi(H) f\left(r, \theta, z, v_{z}, t\right) d z d v_{z}
$$

or, using Eqs. (4) and (6), as

$$
\begin{aligned}
\Psi(r, \theta, t)= & \iint \psi(H) F(r, \theta, \epsilon, t) \\
& \times \frac{\delta(H-\epsilon)}{J(r, \theta, \epsilon,\{\phi\}, t)} d \epsilon d z d v_{z} \\
= & \int \psi(\epsilon) F(r, \theta, \epsilon, t) d \epsilon .
\end{aligned}
$$

The kinetic equation for $F$ can be deduced by comparing the result of two possible different ways of evaluating $\partial \Psi / \partial t$. Using Eq. (9), one can write

$$
\frac{\partial \Psi(r, \theta, t)}{\partial t}=\int \psi(\epsilon) \frac{\partial F(r, \theta, \epsilon, t)}{\partial t} d \epsilon .
$$


Conversely, using definition (8), the time derivative of $\Psi(r, \theta, t)$ can be evaluated as

$$
\frac{\partial \Psi(r, \theta, t)}{\partial t}=\iint\left[\psi(H) \frac{\partial f}{\partial t}+\psi^{\prime}(H) \frac{\partial H}{\partial t} f\right] d z d v_{z},
$$

where $\psi^{\prime}(H)$ indicates $d \psi(H) / d H$. If $\partial f / \partial t$ is expressed using Eq. (1), it follows that

$$
\begin{aligned}
\iint \psi(H) \frac{\partial f}{\partial t} d z d v_{z}= & -\iint \psi(H)\left[\boldsymbol{\nabla}_{\perp} \cdot\left(\frac{\mathbf{e}_{z} \times \boldsymbol{\nabla}_{\perp} \phi}{B_{0}} f\right)\right. \\
& \left.+\frac{\partial\left(v_{z} f\right)}{\partial z}+\frac{\partial}{\partial v_{z}}\left(\frac{e}{m} \frac{\partial \phi}{\partial z} f\right)\right] d z d v_{z}
\end{aligned}
$$

The sum of the last two terms in Eq. (12) vanishes, as an integration by parts shows. Then, being

$$
f \boldsymbol{\nabla} \cdot \mathbf{v}=\boldsymbol{\nabla} \cdot(f \mathbf{v})-\mathbf{v} \cdot \boldsymbol{\nabla} f,
$$

Eq. (12) can be written as

$$
\begin{aligned}
\iint \psi(H) \frac{\partial f}{\partial t} d z d v_{z}= & \iint \boldsymbol{\nabla}_{\perp} \cdot\left(\psi(H) \frac{\mathbf{e}_{z} \times \boldsymbol{\nabla}_{\perp} \phi}{B_{0}} f\right) d z d v_{z} \\
& -\iint \boldsymbol{\nabla}_{\perp} \psi(H) \cdot \frac{\mathbf{e}_{z} \times \boldsymbol{\nabla}_{\perp} \boldsymbol{\phi}}{B_{0}} f d z d v_{z} .
\end{aligned}
$$

The second integral in Eq. (13) vanishes, as $\nabla_{\perp} \psi(H)=$ $-e \psi^{\prime}(H) \nabla_{\perp} \phi$. Using the expressions for $f$ and $G$ given by Eqs. (4) and (6), respectively, Eq. (12) becomes

$$
\begin{aligned}
\iint \psi(H) \frac{\partial f}{\partial t}= & -\nabla_{\perp} \cdot \iiint \psi(H) \frac{\mathbf{e}_{z} \times \boldsymbol{\nabla}_{\perp} \phi}{B_{0}} F(r, \theta, \epsilon, t) \\
& \times \frac{\delta(H-\epsilon)}{J(r, \theta, \epsilon,\{\phi\}, t)} d \epsilon d z d v_{z} \\
= & -\int \psi(\epsilon) \nabla_{\perp} \cdot\left(\mathbf{v}_{D} F\right) d \epsilon,
\end{aligned}
$$

where the velocity $\mathbf{v}_{D}$ has been defined as

$$
\mathbf{v}_{D}(r, \theta, \epsilon, t)=\iint \frac{\mathbf{e}_{z} \times \nabla_{\perp} \phi}{B_{0}} \frac{\delta(H-\epsilon)}{J(r, \theta, \epsilon,\{\phi\}, t)} d z d v_{z}
$$

The second term in Eq. (11) can be evaluated as

$$
\begin{aligned}
\int \psi^{\prime}(H) \frac{\partial H}{\partial t} f d z d v_{z}= & -\iint \psi^{\prime}(H) e \frac{\partial \phi}{\partial t} F(r, \theta, \epsilon, t) \\
& \times \frac{\delta(H-\epsilon)}{J(r, \theta, \epsilon,\{\phi\}, t)} d \epsilon d z d v_{z} \\
= & \int \psi^{\prime}(\epsilon) F(r, \theta, \epsilon, t) v_{\epsilon} d \epsilon \\
= & -\int \psi(\epsilon) \frac{\partial}{\partial \epsilon}\left(F v_{\epsilon}\right) d \epsilon
\end{aligned}
$$

with

$$
v_{\epsilon}(r, \theta, \epsilon, t)=-\iint e \frac{\partial \phi}{\partial t} \frac{\delta(H-\epsilon)}{J(r, \theta, \epsilon,\{\phi\}, t)} d z d v_{z}
$$

Reordering the results of Eqs. (11), (14), and (16) and comparing Eq. (10) with Eq. (11), it follows that

$$
\begin{aligned}
\int \psi(\epsilon) \frac{\partial F}{\partial t}= & -\int \psi(\epsilon) \boldsymbol{\nabla}_{\perp} \cdot\left(\mathbf{v}_{D} F\right) d \boldsymbol{\epsilon} \\
& -\int \psi(\epsilon) \frac{\partial}{\partial \epsilon}\left(v_{\epsilon} F\right) d \epsilon .
\end{aligned}
$$

As Eq. (18) is valid for every choice of the function $\psi(\epsilon)$, one is led to the conclusion that the time derivative of $F$ must satisfy the following equation:

$$
\frac{\partial F}{\partial t}+\nabla_{\perp} \cdot\left(\mathbf{v}_{D} F\right)+\frac{\partial}{\partial \epsilon}\left(v_{\epsilon} F\right)=0
$$

which represents the kinetic equation for the function $F(r, \theta, \epsilon, t)$.

The function $g$, defined as

$$
\begin{aligned}
g(z, r, \theta, \epsilon,\{\phi\}, t) & =\int G\left(z, v_{z}, r, \theta, \epsilon,\{\phi\}, t\right) d v_{z} \\
& =\int \frac{\delta(H-\epsilon)}{J(r, \theta, \epsilon,\{\phi\}, t)} d v_{z} \\
& =\frac{[\epsilon+e \phi(r, \theta, z, t)]^{-1 / 2}}{\int[\epsilon+e \phi(r, \theta, z, t)]^{-1 / 2} d z}
\end{aligned}
$$

can be introduced, and it expresses the distribution of the probability of finding a particle in the axial position $z$. In fact, one can write

$$
\begin{aligned}
g(z, r, \theta, \epsilon, t) \Delta z & =\frac{[e \phi(r, \theta, z, t)+\epsilon]^{-1 / 2} \Delta z}{\int[e \phi(r, \theta, z, t)+\epsilon]^{-1 / 2} d z} \\
& =\frac{\Delta z / v_{z}}{\int d z / v_{z}}=\frac{\Delta t}{\int d t},
\end{aligned}
$$

and $\Delta t / \int d t$ represents the ratio between the time the particle spends moving from $z$ to $z+\Delta z$ and the bouncing period, which corresponds to the probability of finding a particle between $z$ and $z+\Delta z$.

Using the definition (20) for $g$, the streaming velocities (15) and (17) can be expressed as 


$$
\begin{gathered}
\mathbf{v}_{D}(r, \theta, \epsilon, t)=\int \frac{\mathbf{e}_{z} \times \nabla_{\perp} \phi}{B_{0}} g(z, r, \theta, \epsilon,\{\phi\}, t) d z, \\
v_{\epsilon}(r, \theta, \epsilon, t)=-\int e \frac{\partial \phi}{\partial t} g(z, r, \theta, \epsilon,\{\phi\}, t) d z
\end{gathered}
$$

In particular, observing that

$$
\frac{d \epsilon}{d t}=-e \frac{\partial \phi}{\partial t}
$$

and recalling the physical meaning of $g$, Eq. (21), the streaming velocities present in Eq. (19) assume the meaning of bounce-averaged streaming velocities in the axial plane and along the axial-energy coordinate.

The particle density to be used in the Poisson equation can be expressed as

$$
\begin{aligned}
n(r, \theta, z, t) & =\int f\left(r, \theta, z, v_{z}, t\right) d v_{z} \\
& =\int F(r, \theta, \epsilon, t) g(z, r, \theta, \epsilon,\{\phi\}, t) d \epsilon
\end{aligned}
$$

and, consequently, the complete kinetic model for $F(r, \theta, \epsilon, t)$ can be written as

$$
\begin{gathered}
\frac{\partial F}{\partial t}+\nabla_{\perp} \cdot\left(\mathbf{v}_{D} F\right)+\frac{\partial}{\partial \epsilon}\left(v_{\epsilon} F\right)=0, \\
\nabla^{2} \phi=\frac{e}{\varepsilon_{0}} \int F(r, \theta, \epsilon, t) g(z, r, \theta, \epsilon,\{\phi\}, t) d \epsilon, \\
\mathbf{v}_{D}=\int \frac{\mathbf{e}_{z} \times \nabla_{\perp} \phi}{B_{0}} g(z, r, \theta, \epsilon,\{\phi\}, t) d z, \\
v_{\epsilon}=-\int e \frac{\partial \phi}{\partial t} g(z, r, \theta, \epsilon,\{\phi\}, t) d z, \\
g(z, r, \theta, \epsilon,\{\phi\}, t)=\frac{[\epsilon+e \phi(r, \theta, z, t)]^{-1 / 2}}{\int[\epsilon+e \phi(r, \theta, z, t)]^{-1 / 2} d z} .
\end{gathered}
$$

\section{CONSERVATION PROPERTIES}

The kinetic model considered in this paper satisfies some conservation laws. The first property considered here is the conservation of the canonical angular momentum. When the inertia terms are neglected [4], the canonical angular momentum $\mathbb{L}_{z}$ is defined as

$$
\mathbb{L}_{z}=-\frac{e B_{0}}{2} \int_{0}^{R_{w}} \int_{0}^{2 \pi} \int_{-\infty}^{+\infty} r^{2} F(r, \theta, \epsilon, t) r d r d \theta d \epsilon .
$$

Using the kinetic equation (19), the time derivative of $L_{z}$ can be written as

$$
\frac{d L_{z}}{d t}=\frac{e B_{0}}{2} \int_{0}^{R_{w}} \int_{0}^{2 \pi} \int_{-\infty}^{+\infty} r^{2}\left[\nabla_{\perp} \cdot\left(\mathbf{v}_{D} F\right)\right] r d r d \theta d \epsilon
$$

or, equivalently, as

$$
\frac{d L_{z}}{d t}=e \int_{0}^{R_{w}} \int_{0}^{2 \pi} \int_{-\infty}^{+\infty} \int_{-\infty}^{+\infty} F g \mathbf{e}_{\theta} \cdot \nabla \phi r^{2} d r d \theta d \epsilon d z,
$$

having employed the definition (22) for $\mathbf{v}_{D}$ and observing that $\mathbf{v}_{D}\left(R_{w}, \theta\right) \cdot \mathbf{e}_{r}=0$, as the electric field is perpendicular to the surface of the electrodes.

By expressing the integral of $F g$ over $\epsilon$ through the Poisson equation, Eq. (29) becomes

$$
\frac{d \mathbb{L}_{z}}{d t}=\varepsilon_{0} \int \nabla^{2} \phi \frac{\partial \phi}{\partial \theta} d \mathbf{r}=\varepsilon_{0} \oint_{\partial V} \frac{\partial \phi}{\partial \theta} \nabla \phi \cdot \mathbf{n} d A,
$$

in which the identity

$$
\left(\nabla^{2} \phi\right) \frac{\partial \phi}{\partial \theta}=\nabla \cdot\left(\nabla \phi \frac{\partial \phi}{\partial \theta}\right)-\frac{1}{2} \frac{\partial(\nabla \phi)^{2}}{\partial \theta}
$$

and the periodicity of $\phi$ with respect to $\theta$ have been employed. As $\partial \phi / \partial \theta$ vanishes for $r=R_{w}$ ( $\phi$ is constant at each electrode), the conservation law for the canonical angular momentum is readily obtained.

The analysis of the energy conservation law is more subtle. The total energy of the plasma, $\mathbb{E}_{\text {tot }}$, can be expressed as the sum of the kinetic energy $\mathbb{E}_{k i n}$ and of the potential energy of each couple of charged particles, $\mathbb{E}_{\text {pot }}$, where $\mathbb{E}_{\text {kin }}$ and $\mathbb{E}_{\text {pot }}$ are defined as

$$
\begin{aligned}
\mathbb{E}_{k i n}(t)=\frac{1}{2} \iint & \int v_{z}^{2} F(r, \theta, \epsilon, t) \\
\times & G\left(z, v_{z}, r, \theta, \epsilon,\{\phi\}, t\right) d \mathbf{r} d v_{z} d \epsilon, \\
\mathbb{E}_{\text {pot }}(t)= & -\frac{1}{2} \iint e \phi F(r, \theta, \epsilon, t) \\
& \times g(z, r, \theta, \epsilon,\{\phi\}, t) d \mathbf{r} d \epsilon .
\end{aligned}
$$

The quantity $\mathbb{E}_{\text {tot }}$ can also be expressed as

$$
\mathbb{E}_{\text {tot }}=\mathbb{E}_{\epsilon}-\mathbb{E}_{\text {pot }},
$$

where $\mathbb{E}_{\epsilon}$ is

$$
\begin{aligned}
\mathbb{E}_{\epsilon} & =\mathbb{E}_{k i n}+2 \mathbb{E}_{\text {pot }} \\
& =\iint \epsilon F(r, \theta, \epsilon, t) g(z, r, \theta, \epsilon,\{\phi\} t) d \mathbf{r} d \boldsymbol{\epsilon}
\end{aligned}
$$

and the time derivative of the total energy can be evaluated as

$$
\frac{d \mathbb{E}_{t o t}}{d t}=\frac{d \mathbb{E}_{\epsilon}}{d t}-\frac{d \mathbb{E}_{p o t}}{d t}
$$

By integrating along $z$ and using Eq. (19), the time derivative of $\mathbb{E}_{\epsilon}$ becomes 


$$
\begin{aligned}
\frac{d \mathbb{E}_{\epsilon}}{d t} & =\iiint \epsilon \frac{\partial F}{\partial t} r d r d \theta d \epsilon \\
& =-\iiint \epsilon\left[\boldsymbol{\nabla} \cdot\left(\mathbf{v}_{D} F\right)+\frac{\partial}{\partial \epsilon}\left(v_{\epsilon} F\right)\right] r d r d \theta d \epsilon \\
& =\int \oint \epsilon F \mathbf{v}_{D} \cdot \mathbf{n} d l d \epsilon+\iiint v_{\epsilon} F r d r d \theta d \epsilon .
\end{aligned}
$$

As $\mathbf{v}_{D} \cdot \mathbf{e}_{r}$ vanishes on the electrodes, it follows that

$$
\begin{aligned}
\frac{d \mathbb{E}_{\epsilon}}{d t} & =-\iint e \frac{\partial \phi}{\partial t} g F d \mathbf{r} d \epsilon=\frac{\varepsilon_{0}}{2} \int \frac{\partial(\boldsymbol{\nabla} \phi)^{2}}{\partial t} d \mathbf{r} \\
& =\frac{d \mathbb{E}_{e l}}{d t},
\end{aligned}
$$

where $\mathbb{E}_{e l}$ is the energy associated with the electric field, which is defined in the following way:

$$
\mathbb{E}_{e l}=\frac{\varepsilon_{0}}{2} \int(\boldsymbol{\nabla} \phi)^{2} d \mathbf{r}=\frac{\varepsilon_{0}}{2} \int \mathbf{E}^{2} d \mathbf{r} \text {. }
$$

The time derivative of the potential energy $\mathbb{E}_{\text {pot }}$ is evaluated as

$$
\begin{aligned}
\frac{d \mathbb{E}_{p o t}}{d t} & =-\frac{\varepsilon_{0}}{2} \frac{d}{d t} \int \phi \nabla^{2} \phi d \mathbf{r} \\
& =-\frac{\varepsilon_{0}}{2} \frac{d}{d t} \oint \phi \nabla \phi \cdot \mathbf{n} d S+\frac{d \mathbb{E}_{e l}}{d t},
\end{aligned}
$$

where the Poisson equation and the divergence theorem have been used. Thus, from Eq. (36), the time derivative of the total energy becomes

$$
\frac{d \mathbb{E}_{t o t}}{d t}=\frac{\varepsilon_{0}}{2} \frac{d}{d t} \oint \phi \nabla \phi \cdot \mathbf{n} d S .
$$

Equation (41) has a simple physical meaning. In fact, it states that the variation of the plasma total energy equals the power received by the system from the environment: the external energy is necessary for the trap to maintain the electrodes at the fixed potential. As the electric potential is zero for the central electrode, the surface integral appearing in Eq. (41) can be calculated by considering only the region of the side electrodes (at potential $-V$ ), and Eq. (41) can be rewritten as

$$
\frac{d \mathbb{E}_{t o t}}{d t}=\frac{\varepsilon_{0}}{2} \frac{d}{d t}(V \oint \boldsymbol{\nabla} \phi \cdot \mathbf{n}) d S .
$$

According to the Coulomb law for a conductor surface [22], one can write

$$
\boldsymbol{\nabla} \phi \cdot \mathbf{n}=\frac{\sigma}{\varepsilon_{0}},
$$

where $\sigma$ is the surface charge density of the conductor. Introducing this expression in Eq. (42), it follows that

$$
\frac{d \mathbb{E}_{t o t}}{d t}=\frac{1}{2} \frac{d}{d t}(-V q)
$$

which is exactly the power supplied to the end electrodes in order to maintain them at potential $-V$ during the plasma evolution ( $q$ is the total charge of the electrodes).

Finally, from Eqs. (40) and (41), the variation of the kinetic energy $\mathbb{E}_{k i n}$ associated with the particles can be evaluated as

$$
\frac{d \mathbb{E}_{k i n}}{d t}=\varepsilon_{0} \frac{d}{d t} \oint \phi \nabla \phi \cdot \mathbf{n} d S-\frac{d \mathbb{E}_{e l}}{d t} .
$$

\section{CONNECTIONS WITH THE FLUID MODEL}

Starting from the kinetic model presented here, the fluid model for non-neutral plasmas that was introduced by Finn et al. [12,13] and refined by Coppa et al. [11] can be deduced. Moreover, through the present kinetic model, the meaning of the energy conservation law found within the fluid model in Ref. [11] can be pointed out.

According to Finn et al.'s model, charged particles in a Penning trap are regarded as strings of variable length (and, consequently, of variable density) in which the MaxwellBoltzmann distribution is reached along the $z$ direction $[11,17]$. This means that the electron density $n(r, \theta, z, t)$, which is related to the kinetic distribution function $F(r, \theta, \epsilon, t)$ through Eq. (25), can be written as

$$
n(r, \theta, z, t)=\mathcal{N}(r, \theta, t) \exp \left[\frac{e \phi(r, \theta, z, t)}{k_{B} T}\right],
$$

where $T$ is the electron temperature and $\mathcal{N}$ is a function independent of $z$. The fluid equation for the $z$-integrated density $\sigma(r, \theta, t)$, defined as

$$
\sigma(r, \theta, t)=\int n(r, \theta, z, t) d z
$$

can be written as in Ref. [11]:

$$
\frac{\partial \sigma}{\partial t}+\nabla_{\perp} \cdot\left(\mathbf{V}_{\perp} \sigma\right)=0
$$

where $\mathbf{V}_{\perp}$ is defined in the following way [11]:

$$
\mathbf{V}_{\perp}=\frac{1}{B_{0}} \mathbf{e}_{z} \times \frac{\int \exp \left[(e \phi) /\left(k_{B} T\right)\right] \nabla_{\perp} \phi d z}{\int \exp \left[(e \phi) /\left(k_{B} T\right)\right] d z}
$$

Assuming that the energy dependence of the $z$-integrated distribution function $F$ is factorized as

$$
F(r, \theta, \epsilon, t)=\frac{\mathcal{F}(r, \theta, t)}{\sqrt{k_{B} T}} \exp \left(-\frac{\epsilon}{k_{B} T}\right) \int \frac{d z}{\sqrt{e \phi+\epsilon}},
$$


the assumption (46) is recovered and the fluid equation (48) is deduced, from the kinetic model developed here. In fact, within this hypothesis, recalling Eq. (25), the particle density can be written as

$$
\begin{aligned}
n(r, \theta, z, t) & =\frac{\mathcal{F}(r, \theta, t)}{\sqrt{k_{B} T}} \int \frac{\exp \left[-\epsilon /\left(k_{B} T\right)\right]}{\sqrt{e \phi+\epsilon}} d \epsilon \\
& =\sqrt{\pi} \mathcal{F}(r, \theta, t) \exp \left[\frac{e \phi(r, \theta, z, t)}{k_{B} T}\right],
\end{aligned}
$$

and by simply imposing the relation $\sqrt{\pi} \mathcal{F}(r, \theta, t)$ $=\mathcal{N}(r, \theta, t)$ between the functions $\mathcal{F}$ and $\mathcal{N}$, the assumption (46) is readily obtained.

The fluid equation (48) can be deduced from the kinetic model by integrating Eq. (19) over the energy $\epsilon$. In fact, requiring $F$ to have the form expressed in Eq. (50) and recalling the results of the integral (51) and the relation between $\mathcal{F}$ and $\mathcal{N}$, the fluid equation (48) is obtained.

It must be pointed out that the assumption (46) implies that the electrons are distributed as stated by the canonical distribution [21] in the phase space $\left(z, v_{z}\right)$, for each planar position $(r, \theta)$ and, in fact, it is possible to write

$$
f\left(r, \theta, z, v_{z}, t\right)=\sqrt{\frac{m}{2 k_{B} T}} \mathcal{F}(r, \theta, t) \exp \left(-\frac{H}{k_{B} T}\right),
$$

as follows from Eqs. (4) and (50).

In Ref. [11], the energy conservation of the fluid model was investigated and a conservation law was found in this context. The kinetic theory makes it possible to discuss the energy conservation in more detail. As the fluid model is obtained having fixed the energy dependence of the distribution function, the energy of the particles cannot vary in time self-consistently as prescribed by Eq. (24). There are two main consequences of this fact. The first concerns the kinetic energy of the plasma. The energy $\mathbb{E}_{\epsilon}$ can be evaluated directly from Eq. (35) by using the assumption (50): one obtains

$$
\begin{aligned}
\mathbb{E}_{\epsilon}= & \frac{1}{\sqrt{k_{B} T}} \iint \epsilon \frac{\exp \left[-\epsilon /\left(k_{B} T\right)\right]}{\sqrt{e \phi+\epsilon}} \mathcal{F}(r, \theta, t) d \epsilon d \mathbf{r} \\
= & \frac{1}{\sqrt{k_{B} T}} \int \exp \left(\frac{e \phi}{k_{B} T}\right) \mathcal{F}(r, \theta, t) \\
& \times \int \epsilon \frac{\exp \left[-(\epsilon+e \phi) /\left(k_{B} T\right)\right]}{\sqrt{e \phi+\epsilon}} d \epsilon d \mathbf{r} .
\end{aligned}
$$

The integration over $\epsilon$ can be performed analytically, so that the energy $\mathbb{E}_{\epsilon}$ can be written as

$$
\begin{aligned}
\mathbb{E}_{\epsilon} & =\int\left(\frac{1}{2} k_{B} T-e \phi\right) \exp \left(\frac{e \phi}{k_{B} T}\right) \mathcal{N}(r, \theta, t) d \mathbf{r} \\
& =\frac{1}{2} \mathbb{N} k_{B} T-e \int \phi(r, \theta, z, t) n(r, \theta, z, t) d \mathbf{r} \\
& =\text { const }+2 \mathbb{E}_{\text {pot }},
\end{aligned}
$$

with $\mathbb{N}$ denoting the (constant) number of particles in the trap. Equation (54) leads to

$$
\frac{d}{d t}\left(\mathbb{E}_{\varepsilon}-2 \mathbb{E}_{p o t}\right)=0
$$

and, as a consequence, from Eq. (35), it follows that

$$
\frac{d \mathbb{E}_{k i n}}{d t}=0
$$

within the fluid model, in contrast to Eq. (45). Equation (56) has a simple physical meaning: it indicates that Eq. (46) describes the plasma as a sequence of thermal equilibrium states, fixing the kinetic energy of the plasma particles [see Eq. (52)] and, thus, neglecting its variation.

The second consequence concerns the conservation of the total energy of the plasma, $\mathbb{E}_{t o t}$. As Eq. (24) is not satisfied within the fluid model, there is no exchange between potential and plasma particle energy, to enable preservation of the total amount of energy in the system. In particular, from Eq. (54) and considering that Eq. (40) still holds, one obtains

$$
\frac{d \mathbb{E}_{t o t}}{d t}=\frac{d \mathbb{E}_{e l}}{d t}-\frac{\varepsilon_{0}}{2} \frac{d}{d t} \oint \phi \nabla \phi \cdot \mathbf{n} d S
$$

within the fluid model, unlike Eq. (41).

In the particular case in which, during the evolution, it is true that $d \mathbb{E}_{k i n} / d t=0$ or, equivalently [see Eq. (45)], if the condition

$$
\frac{d \mathbb{E}_{e l}}{d t}=\varepsilon_{0} \frac{d}{d t} \oint \phi \nabla \phi \cdot \mathbf{n} d S
$$

holds, then the conservation of the total energy of the plasma is found within the fluid model, as well. In fact, if Eq. (58) is verified, Eq. (57) reduces to Eq. (41) or, in other terms, the fluid model states a correct energy conservation law.

In Ref. [11] [Eq. (35), p. 1136], the quantity $\mathbb{E}_{\text {eff }}$ was introduced as a guess to express the total energy of the plasma. In particular, the following conservation property was found [see Ref. 11, Eq. (41), p. 1136]:

$$
\frac{d \mathbb{E}_{e f f}}{d t}=\frac{1}{2} \frac{d \mathbb{E}_{e l}}{d t} .
$$

Of course, Eq. (59) cannot express a conservation in the form of Eq. (41) in a general plasma evolution: this would transcend the fluid model. However, if Eq. (58) holds, the energy conservation law (59) found in Ref. [11] yields 


$$
\frac{d \mathbb{E}_{e f f}}{d t}=\frac{\varepsilon_{0}}{2} \frac{d}{d t} \oint \phi \nabla \phi \cdot \mathbf{n} d S,
$$

which is a correct energy conservation law for the quantity $\mathbb{E}_{\text {eff }}$. This means that the energy $\mathbb{E}_{\text {eff }}$ is a correct guess for the total plasma energy because, in the limiting cases where, also within the fluid model, the plasma evolution is such that the total energy of the particles is conserved, Eq. (59) reduces to the form of the general conservation law Eq. (41).

\section{GENERAL PROPERTIES OF THE $z$-INTEGRATED DISTRIBUTION}

There are different ways of expressing the distribution $F(r, \theta, \epsilon, t)$ as a function of more common physical quantities. First, the function $F(r, \theta, \epsilon, t)$ is tightly related to experimental data. Usually, experiments regarding non-neutral plasmas [16] provide the $z$-integrated distribution of the particles, $\sigma(r, \theta, t)$ [defined in Eq. (47)], and the value of the kinetic energy of the particles, $s$, through the distribution $p_{K}(\mathrm{~s}, r, \theta, t)$. The distribution function $p_{K}$ is normalized so that $\int p_{K}(\varsigma, r, \theta, t) d \varsigma=1$

The kinetic energy $s$ can be expressed as

$$
\varsigma=\epsilon+e \phi_{m},
$$

where $\phi_{m}$ is the potential of the point where the kinetic energy of the particles is measured and, as a consequence, the distribution function $F(r, \theta, \epsilon, t)$ can be written directly from experimental data as

$$
F(r, \theta, \epsilon, t)=\sigma(r, \theta, t) p_{K}\left(\epsilon+e \phi_{m}, r, \theta, t\right) .
$$

Moreover, in analogy with Refs. [11-13], where the plasma density $n_{c}(r, \theta, t)$ at the center of the trap $(z=0)$ is supposed to be known, in the present kinetic model it is possible to fix the distribution function $f\left(r, \theta, z, v_{z}, t\right)$ at the center of the trap, in the form

$$
f\left(r, \theta, z=0, v_{z}, t\right)=n_{c}(r, \theta, t) p_{v}\left(v_{z}, r, \theta, t\right),
$$

with $p_{v}$ expressing the $z$-velocity distribution of the particle in the planar position $(r, \theta)$, where $\int p_{v}\left(v_{z}, r, \theta, t\right) d v_{z}=1$. The corresponding distribution function $F(r, \theta, \epsilon, t)$ can be expressed as

$$
\begin{aligned}
F(r, \theta, \epsilon, t)= & n_{c}(r, \theta, t) p_{v}\left(\sqrt{\frac{2\left(\epsilon+e \phi_{c}\right)}{m}}, r, \theta, t\right) \\
& \times \int \sqrt{\frac{2}{m(\epsilon+e \phi)}} d z,
\end{aligned}
$$

where the potential $\phi_{c}(r, \theta, t)$ denotes the potential of the trap at $z=0$ and can be evaluated by solving the Poisson equation

$$
\nabla^{2} \phi_{c}=\frac{e}{\varepsilon_{0}} n_{c}(r, \theta, t)
$$

in an axially infinite cylinder.

Finally, in Refs. [11-13], the plasma length $\mathcal{L}(r, \theta, t)$ was introduced, in order to express $\sigma(r, \theta, t)$ as $n_{c}(r, \theta, t) \mathcal{L}(r, \theta, t)$. In analogy with the fluid model, a plasma length that depends on the axial energy of the particles is introduced to express the distribution function $F(r, \theta, \epsilon, t)$ as

$$
F(r, \theta, \epsilon, t)=n_{c}(r, \theta, t) \mathcal{L}(r, \theta, \epsilon, t)
$$

and, using Eq. (64), $\mathcal{L}(r, \theta, \epsilon, t)$ can be written as

$$
\mathcal{L}(r, \theta, \epsilon, t)=\sqrt{2} \int \frac{p_{v}\left(\epsilon+e \phi_{c}, r, \theta, t\right)}{\sqrt{m(\epsilon+e \phi)}} d z .
$$

\section{EQUILIBRIUM STATES}

As can be readily verified, any axially symmetric distribution function of the form $F=F(r, \epsilon)$, associated with the potential $\phi=\phi(r, z)$ given by the self-consistent Poisson equation

$$
\nabla^{2} \phi(r, z)=\frac{e}{\epsilon_{0}} \int \frac{F(r, \epsilon)[e \phi(r, z)+\epsilon]^{-1 / 2}}{\int[e \phi(r, z)+\epsilon]^{-1 / 2} d z} d \epsilon,
$$

represents an equilibrium distribution.

The stationary states of the plasma have been investigated by solving numerically the Poisson equation (68) for different choices of the function $F(r, \epsilon)$. Equation (68) is a nonlinear elliptic partial differential equation in two dimensions. Discretizing the variables $r, z$, and $\epsilon$ and using suitable quadrature formulas to evaluate the $z$ and $\epsilon$ integrals [23], the Poisson equation (68) can be solved numerically by adding the term $\partial \phi / \partial t$ to its left-hand side and seeking the steady state of the time-dependent diffusionlike equation obtained. A time-implicit method has been used to solve the diffusionlike equation. Once $\phi(r, z)$ is evaluated, the density of electrons in the Penning trap is readily found as

$$
n(r, z)=\int \frac{F(r, \epsilon)[e \phi(r, z)+\epsilon]^{-1 / 2}}{\int[e \phi(r, z)+\epsilon]^{-1 / 2} d z} d \epsilon
$$

and also the bounce-averaged drift velocity $\mathbf{v}_{D}=v_{D, \theta} \mathbf{e}_{\theta}$ can be evaluated as a function of $r$ and $\epsilon$. 

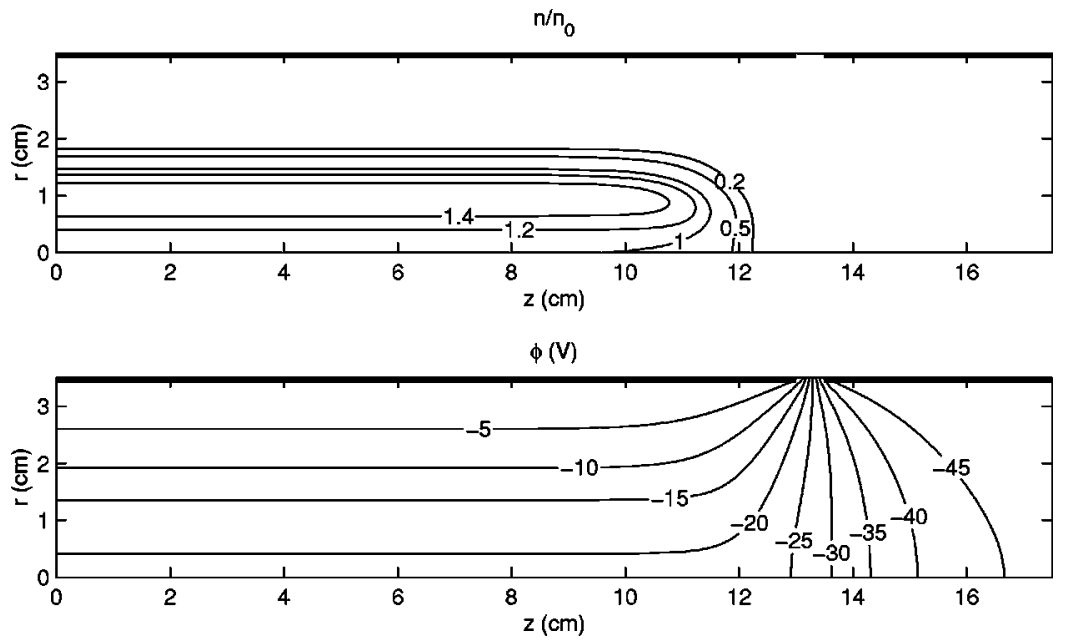

FIG. 1. Equilibrium plasma density and potential for a typical Penning trap. The confining potential is $V=-50 \mathrm{~V}$, the central electrode length $L_{c}=26 \mathrm{~cm}$, the end electrode length $L_{s}$ $=4 \mathrm{~cm}$, the gap length $L_{g}=0.5 \mathrm{~cm}$, the wall radius $R_{w}=3.5 \mathrm{~cm}$, the plasma temperature $T$ $=1000 \mathrm{~K}$, and the magnetic field $B_{0}=1 \mathrm{~T}$. The velocity distribution of the electrons is Maxwellian. The central profile parameters are $n_{0}=5$ $\times 10^{12} \mathrm{~m}^{-3}, \mu=5$, and $r_{p}=2 \mathrm{~cm}$.
The equilibrium solutions have been studied having fixed the plasma density at the center of the trap $n_{c}(r)$ in the form $[5,12,13]$

$n_{c}(r)= \begin{cases}n_{0}\left[1-\left(\frac{r}{r_{p}}\right)^{2}\right]^{2}\left[1+(\mu+2)\left(\frac{r}{r_{p}}\right)^{2}\right] & \text { if } r \leqslant r_{p} \\ 0 & \text { otherwise }\end{cases}$

where the dimensionless positive parameter $\mu$ measures the degree of hollowness of the profile. Different density profiles for different values of the parameter $\mu$ are plotted in Ref. [11]. In particular, the density profile used in all the numerical integrations of the present paper corresponds to $\mu=5$.

The equilibrium potential and the equilibrium plasma density for a standard Penning trap are represented in Fig. 1.
The electron velocity distribution at $z=0$ [see Eq. (63) $]$ is assumed to be Maxwellian, being

$$
p_{v}\left(v_{z}, r\right)=\sqrt{\frac{m}{2 \pi k_{B} T}} \exp \left(-\frac{m v_{z}^{2}}{2 k_{B} T}\right) .
$$

As expected, Fig. 1 shows that the electron density decreases sharply approaching the end electrodes, while the $z$ dependence of the plasma distribution in the grounded central electrode is negligible.

The equilibrium solutions have also been computed in the case of distributions with the form expressed by a truncated Maxwellian, that was recently employed by Hilsabeck and O’Neil [10]:
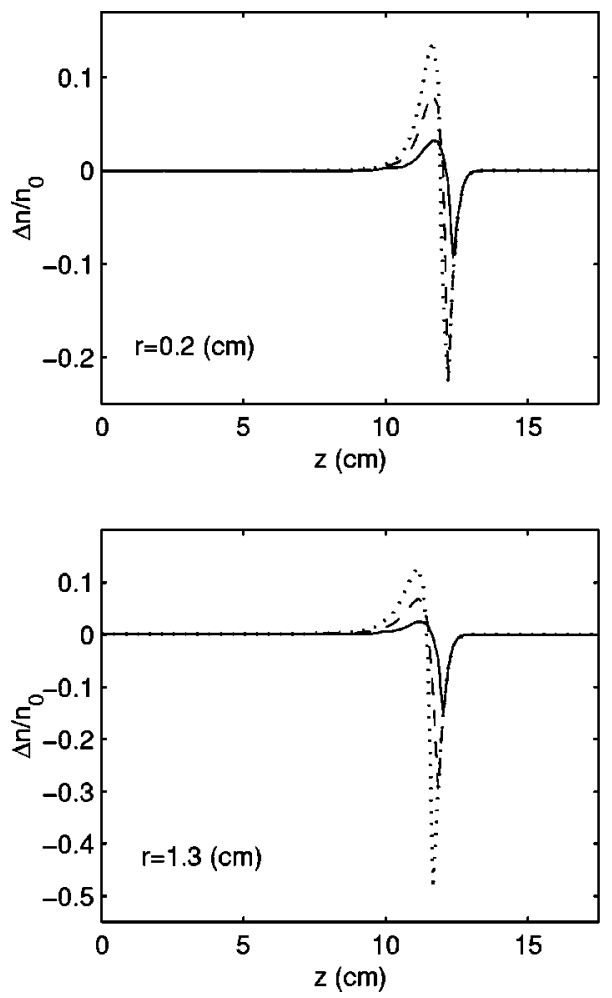
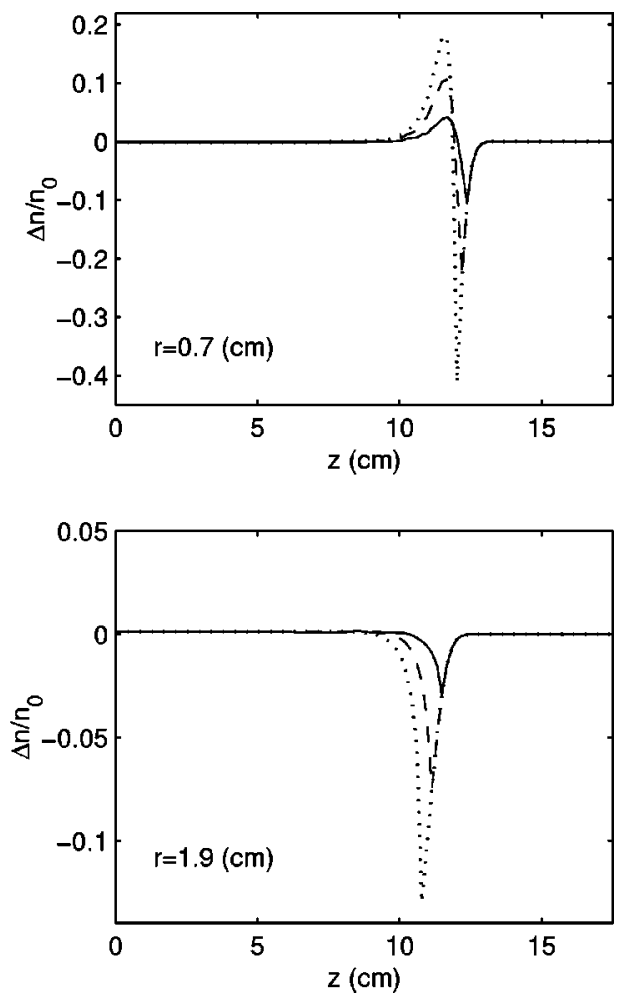

FIG. 2. Plasma density difference $\Delta n / n_{0}$ with respect to the Maxwellian distribution, for a Maxwellian distribution truncated at the energy $S=k_{B} T / 2$ (dotted), $S=k_{B} T$ (dashed), and $S=2 k_{B} T$ (solid). The trap parameters are the same as in Fig. 1. 

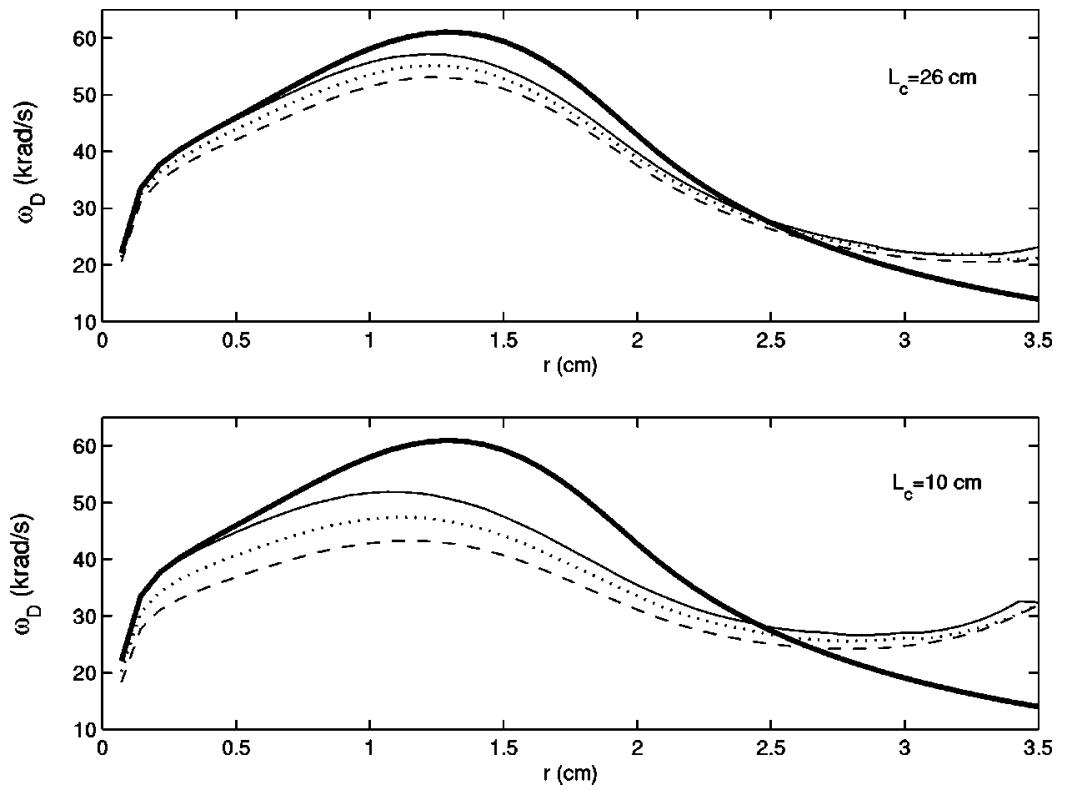

FIG. 3. Bounce-averaged drift frequency $\omega_{D}$ (in $\mathrm{krad} / \mathrm{s}$ ) for electrons at different axial energies: $\epsilon=20.5 \mathrm{eV}$ (solid), $\epsilon=23.3 \mathrm{eV}$ (dotted), and $\epsilon=26 \mathrm{eV}$ (dashed) for two different values of $L_{c}$. The prediction of the two-dimensional theory is shown by the thick solid line. The other trap parameters are the same as in Fig. 1.

$$
p_{v}\left(v_{z}, r\right)=\left\{\begin{array}{l}
\sqrt{\frac{m}{2 \pi k_{B} T}} \exp \left(-\frac{m v_{z}^{2}}{2 k_{B} T}\right) \\
\quad \times\left[\operatorname{erf}\left(\sqrt{\frac{S}{k_{B} T}}\right)\right]^{-1} \text { if } \frac{1}{2} m v_{z}^{2}>S(r), \\
0 \text { otherwise, }
\end{array}\right.
$$

and the corresponding equilibrium potentials and equilibrium electron densities, for different values of $S$, have been compared with the pure Maxwellian case.

Figure 2 compares the differences in the plasma density, with respect to the Maxwellian case, for different truncated Maxwellians at different radii. The difference of the plasma density is more evident at the border of the plasma and depends on $r$. It increases as the Maxwellian distribution is truncated more and more at low energy: in particular, Fig. 2 reveals that the lack of energetic electrons in the truncated Maxwellians implies a shorter penetration length of the plasma into the confining potential wall.

Figures 3 and 4 discuss the azimuthal component of the bounce-averaged drift frequency $\omega_{D}$. In particular, Fig. 3 shows the bounce-averaged drift frequency at different axial energies, compared to the predictions of the classic, twodimensional theory for a short and a long Penning trap. Figure 4 shows the contour plot of the bounce-averaged drift frequency versus the radius and the axial energy of the particles. As was argued $[16,17]$, the drift velocity strongly depends on the particle energy.

\section{CONCLUSIONS}

In the present work, a noncollisional kinetic model for a non-neutral plasma in a Penning trap has been developed describing the evolution of the $z$-integrated distribution function $F(r, \theta, \epsilon, t)$. The general properties of the model have been discussed, in particular the conservation properties. It has been pointed out that the model conserves both the angular momentum of the plasma and the energy of the system. From the kinetic equation, the fluid model introduced by Finn et al. [12,13] and developed by Coppa et al. [11] has been deduced. The distribution function $F(r, \theta, \epsilon, t)$ has also been related to experimentally measured data through Eq. (62). Numerical results have been shown concerning the equilibrium states of the plasma.

Further investigations regarding non-neutral plasma dynamics can be performed using the kinetic model presented here. In particular, the kinetic equation can be linearized in order to study the temporal evolution of an initial perturbation. An eigenvalue equation can be obtained in order to study the spatial and energetic distributions of the modes and, through its spectrum, their frequencies and growth rates. Of particular interest is the mode $m_{\theta}=1$, whose stability

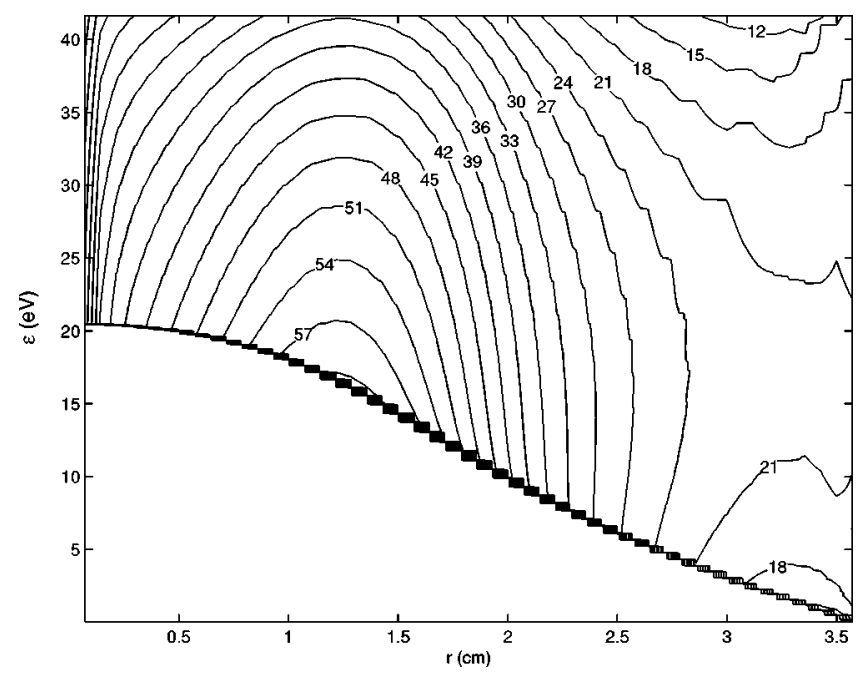

FIG. 4. Contour plot of the bounce-averaged drift frequency $\omega_{D}$ (in $\mathrm{krad} / \mathrm{s}$ ) for electrons at different axial energies and different radii, for the case of a typical Penning trap: the trap parameters are the same as in Fig. 1. 
investigation still represents a challenging problem in the physics of non-neutral plasmas. In comparison to fluid models, the linear analysis performed using the present kinetic model takes into account that particles with different axial energies have different rotation frequencies, and a spread in the axial energies produces a broadening of the unstable mode's resonance with the plasma rotation [10].

The evaluation of the averaged streaming velocities can also be suitably used in order to study, through particle-in- cell codes, the importance of the energy dependence for the evolution of the vortices in a Penning trap.

\section{ACKNOWLEDGMENT}

This work was performed with partial financial support by GNFM (the Italian National Group of Mathematical Physics).
[1] J.H. Malberg and J.S. de Grassie, Phys. Rev. Lett. 35, 577 (1975).

[2] R.C. Davidson, Theory of Nonneutral Plasmas (Benjamin, Reading, MA, 1974).

[3] C.F. Driscoll and K.S. Fine, Phys. Fluids B 2, 1359 (1990).

[4] R.C. Davidson, An Introduction to the Physics of Non-Neutral Plasmas (Addison-Wesley, Redwood City, CA, 1990).

[5] R.C. Davidson and G.M. Felice, Phys. Plasmas 5, 3497 (1998).

[6] R.H. Levy, Phys. Fluids 8, 1288 (1965); 11, 920 (1968).

[7] R.J. Briggs, J.D. Daugherty, and R.H. Levy, Phys. Fluids 13, 421 (1970).

[8] R.A. Smith and M.N. Rosenbluth, Phys. Rev. Lett. 64, 649 (1990).

[9] A.A. Kabantsev and C.F. Driscoll, in Non-Neutral Plasma Physics III, edited by J. J. Bollinger, R. L. Spencer, and R.C. Davidson, AIP Conf. Proc. No. 498 (AIP, Melville, NY, 1999), p. 208.

[10] T.J. Hilsabeck and T.M. O’Neil, Phys. Plasmas 8, 407 (2001).

[11] G.G. M Coppa, A. d'Angola, G.L. Delzanno, and G. Lapenta, Phys. Plasmas 8, 1133 (2001).

[12] J.M. Finn, D. del Castillo-Negrete, and D.C. Barnes, Phys. Plasmas 6, 3744 (1999).
[13] J.M. Finn, D. del Castillo-Negrete, and D.C. Barnes, Phys, Rev. Lett. 84, 2401 (2000).

[14] S.A. Prasad and T.M. O’Neil, Phys. Fluids 22, 278 (1979).

[15] A.J. Peurrung and J. Fajans, Phys. Fluids B 2, 693 (1990).

[16] A.J. Peurrung and J. Fajans, Phys. Fluids B 5, 4295 (1993).

[17] D.H.E. Dubin and T.M. O’Neil, Phys. Plasmas 5, 1305 (1998).

[18] G.G.M. Coppa and P. Ricci, in Non-Neutral Plasma Physics $I V$, edited by F. Anderegg, C. F. Driscoll, and L. Schweikhard, AIP Conf. Proc. No. 606 (AIP, Melville, NY, 2002), pp. 531536.

[19] C.F. Driscoll, Phys. Rev. Lett. 64, 645 (1990).

[20] H. Goldstein, Classical Mechanics (Addison-Wesley, Reading, MA, 1980).

[21] R. Balescu, Equilibrium and Non Equilibrium Statistical Mechanics (Wiley, New York, 1975), pp. 695-727.

[22] J.D. Jackson, Classical Electrodynamics (Wiley, New York, 1975).

[23] Handbook of Mathematical Functions with Formulas, Graphs and Mathematical Tables, edited by M. Abramowitz and I.A. Stegun, Natl. Bur. Stand. Appl. Math. Ser. No. 55 (U.S. GPO, Washington, DC, 1964), p. 889. 